\title{
$s$-Wave Order Parameter in the Presence of a Momentum-Dependent Impurity Scattering Potential
}

\author{
D. Rudzińska, P. Pisarski And G. Harań \\ Institute of Physics, Politechnika Wrocławska \\ Wybrzeże Wyspiańskiego 27, 50-370 Wrocław, Poland
}

\begin{abstract}
Momentum dependence of the impurity potential leads to the anisotropy of a local density of states in isotropic $s$-wave superconductors. We complete this result by discussing the influence of a momentum-dependent impurity potential on the s-wave order parameter. The scattering process is considered in the T-matrix approximation, and the model impurity potential consists of two separate terms representing isotropic (on-site) and anisotropic (momentum-dependent) parts of the potential. We show that the effect of the isotropic part on the order parameter is local whereas the influence of the momentum-dependent term is nonlocal.
\end{abstract}

PACS numbers: 74.62.Dh, 74.78.--w, 74.81.--g

\section{Introduction}

Lowered symmetry of the impurity potential realized by a momentum dependence of the scattering probability has impact on the local properties of superconductors in the immediate vicinity of the impurity [1-3]. Although nonmagnetic impurities do not change thermodynamic properties of an $s$-wave superconducting state [4], their influence can be distinguished in the local density of states or its Fourier transform $[3,5]$, as well as in the local change of the order parameter [6-9]. The nonmagnetic impurity effect on the $s$-wave order parameter has been discussed for point-like [6-9] and spherical [6] model scattering potentials. We complete these studies by including momentum dependence of the impurity potential and consider the evolution of a two-dimensional $s$-wave order parameter around such a scattering center. We perform our calculations in the particle-hole space using $\hat{\tau}_{i}(i=1,2,3)$ notation for the Pauli matrices, and $\hat{\tau}_{0}$ for the identity matrix.

\section{Momentum-dependent impurity potential}

A momentum-dependent impurity potential is assumed in a separable form [10]: 


$$
\hat{v}\left(\boldsymbol{k}, \boldsymbol{k}^{\prime}\right)=\left[v_{\mathrm{i}}+v_{\mathrm{a}} f(\boldsymbol{k}) f\left(\boldsymbol{k}^{\prime}\right)\right] \hat{\tau_{3}},
$$

where $v_{\mathrm{i}}, v_{\mathrm{a}}$ are potential amplitudes in the isotropic and anisotropic channels, respectively. We use a convenient $\alpha$ parameter which divides the scattering strength between these two channels $v_{\mathrm{i}}=\alpha v_{0}, v_{\mathrm{a}}=(1-\alpha) v_{0}$, where $0 \leq \alpha \leq 1$ and $v_{0}$ is a total potential amplitude. $\alpha=1$ corresponds to isotropic and $\alpha=0$ to a fully anisotropic scattering. We study the effect of the impurity potential of a tetragonal symmetry defined by the anisotropy function $f(\boldsymbol{k})=\operatorname{sgn}(\cos 2 \varphi)$, where $\varphi$ is a polar angle of the wave vector $\boldsymbol{k}$.

\section{T-matrix approximation}

We consider a strong scattering regime and apply the T-matrix approach in a way appropriate for a single impurity

$$
\hat{T}\left(\boldsymbol{k}, \boldsymbol{k}^{\prime}, \omega\right)=\hat{v}\left(\boldsymbol{k}, \boldsymbol{k}^{\prime}\right)+\sum_{\boldsymbol{k}^{\prime \prime}} \hat{v}\left(\boldsymbol{k}, \boldsymbol{k}^{\prime \prime}\right) \hat{G}_{0}\left(\boldsymbol{k}^{\prime \prime}, \omega\right) \hat{T}\left(\boldsymbol{k}^{\prime \prime}, \boldsymbol{k}^{\prime}, \omega\right),
$$

where $\hat{G_{0}}(\boldsymbol{k}, \omega)=\left(\omega \hat{\tau_{0}}-\xi_{k} \hat{\tau_{3}}-\Delta_{0} \hat{\tau_{1}}\right)^{-1}$ is the retarded Green function of a uniform $s$-wave superconductor with the order parameter $\Delta_{0}$ and $\xi_{k}=k^{2} / 2 m-\varepsilon_{\mathrm{F}}$ is the quasiparticle energy in the normal state with the Fermi energy $\varepsilon_{\mathrm{F}}$. We take $\hbar=1$. Because of a separable form of the impurity potential (1) the T-matrix reads

$$
\hat{T}\left(\boldsymbol{k}, \boldsymbol{k}^{\prime}, \omega\right)=\hat{T}_{0}(\omega)+\hat{T}_{1}(\omega) f(\boldsymbol{k}) f\left(\boldsymbol{k}^{\prime}\right)+\hat{T}_{2}(\omega) f(\boldsymbol{k})+\hat{T}_{3}(\omega) f\left(\mathbf{k}^{\prime}\right) .
$$
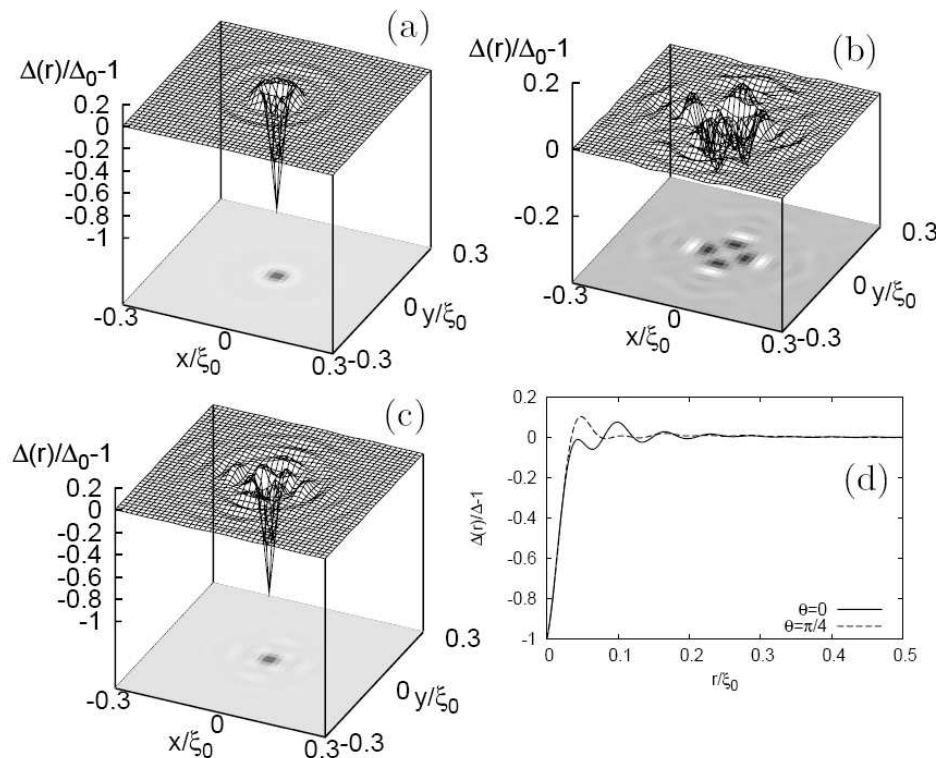

Fig. 1. Local change of the $s$-wave order parameter around a unitary impurity $(c=0)$ at the origin induced by (a) isotropic scattering channel, (b) anisotropic scattering channel, (c) momentum-dependent impurity potential, (d) cuts of (c) for [1,0] and [1,1] directions. The distance from the impurity is in the coherence length $\xi_{0}$ units. 
For the $s$-wave superconductor we obtain a simple solution of Eq. (2):

$$
\hat{T}_{0}(\omega)=\left(1-v_{\mathrm{i}} \hat{\tau}_{3} \hat{G}_{0}\right)^{-1} v_{\mathrm{i}} \hat{\tau}_{3}, \quad \hat{T}_{1}(\omega)=\left(1-v_{\mathrm{a}} \hat{\tau}_{3} \hat{G}_{0}\right)^{-1} v_{\mathrm{a}} \hat{\tau}_{3},
$$

and $\hat{T}_{2}(\omega)=\hat{T}_{3}(\omega)=0$. The T-matrix gives the impurity-induced change of the spatial Green function

$$
\delta \hat{G}(\boldsymbol{r}, \omega)=\sum_{\boldsymbol{k}, \boldsymbol{k}^{\prime}} \mathrm{e}^{\mathrm{i}\left(\boldsymbol{k}-\boldsymbol{k}^{\prime}\right) \boldsymbol{r}} \hat{G}_{0}(\boldsymbol{k}, \omega) \hat{T}\left(\boldsymbol{k}, \boldsymbol{k}^{\prime}\right) \hat{G}_{0}\left(\boldsymbol{k}^{\prime}, \omega\right),
$$

whose off-diagonal element determines the evolution of the order parameter in the vicinity of the impurity

$$
\Delta(\boldsymbol{r})-\Delta_{0}=-g \int_{-\omega_{\mathrm{C}}}^{\omega_{\mathrm{C}}} \frac{\mathrm{d} \omega}{2 \pi} \delta G_{12}(\boldsymbol{r}, \omega),
$$

where $g>0$ is an $s$-wave coupling constant and $\omega_{\mathrm{C}}$ is a cut-off energy.

\section{Results}

We have evaluated the position-dependent order parameter in the vicinity of the impurity for the momentum-dependent scattering potential (1) of a tetragonal
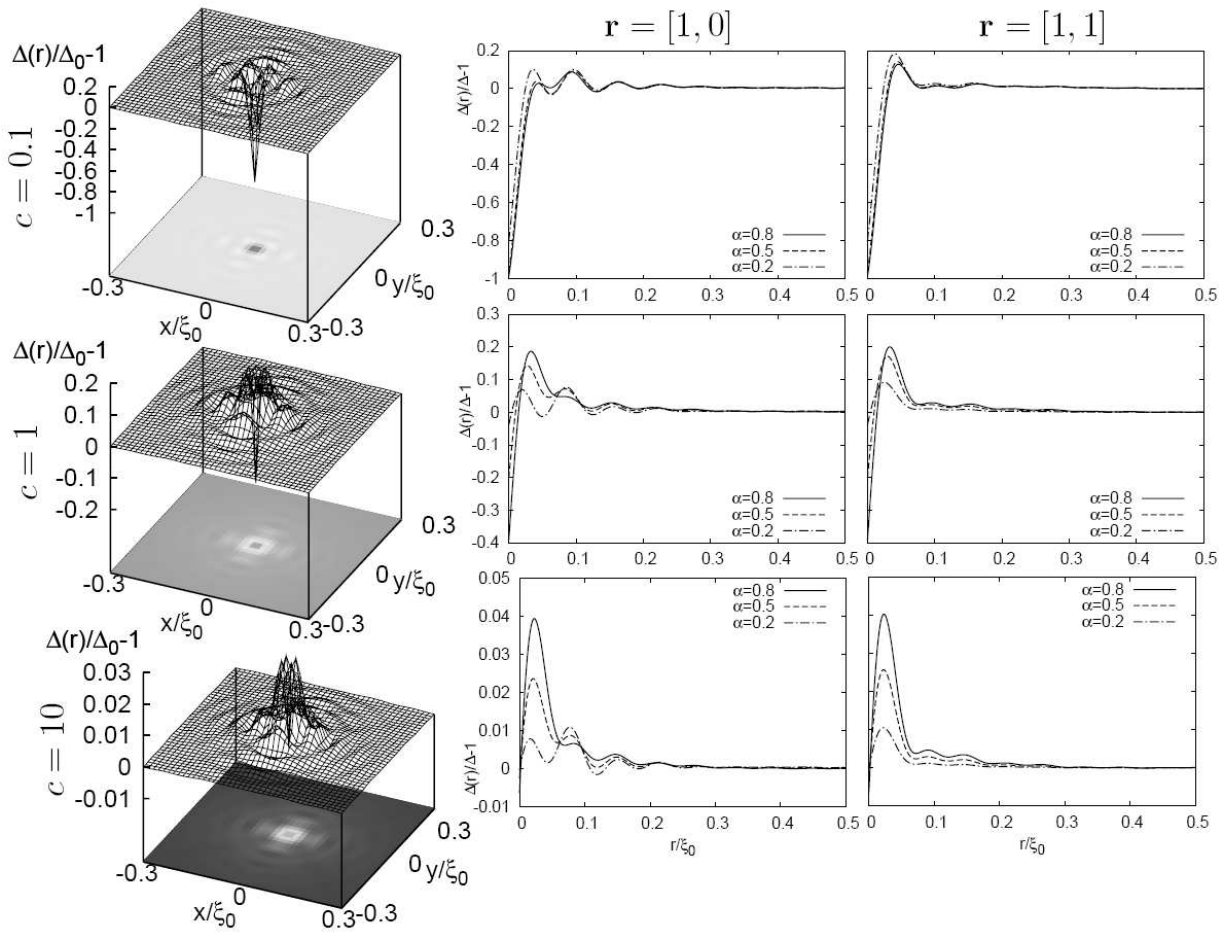

Fig. 2. Evolution of the $s$-wave order parameter around the impurity (first column), $\Delta(\boldsymbol{r})$ for $\boldsymbol{r}=[1,0]$ (second column) and for $\boldsymbol{r}=[1,1]$ (third column) for a finite scattering strength (from the top) $c=0.1,1.0,10$ and the potential partition $\alpha=0.8, \alpha=0.5$, $\alpha=0.2$. 
symmetry. In Fig. 1 we show the evolution of the order parameter around a unitary impurity. The effect of a sole isotropic part of the impurity potential is presented in Fig. 1a while the influence of a momentum-dependent channel is shown in Fig. 1b. We note that the isotropic potential leads to a strong on-site order parameter suppression, whereas the changes induced by the anisotropic channel are nonlocal. The impact of a full anisotropic impurity scattering potential (1) on the $s$-wave order parameter in the unitary limit along with $\Delta(\boldsymbol{r})$ plots for $[1,0]$ (polar angle $\Theta=0$ ) and $[1,1](\Theta=\pi / 4)$ directions are shown in Figs. 1c, d. We also present in Fig. 2 a local effect of a finite impurity potential on the $s$-wave order parameter. In the numerical computations the cut-off energy in the gap equation $\omega_{\mathrm{C}} / \Delta_{0}=18$, and the Fermi energy $\varepsilon_{\mathrm{F}} / \Delta_{0}=12 \pi$ were applied. The results are plotted in $g N_{0}$ units, where $N_{0}=m / 2 \pi$ is the normal state density of states per spin at the Fermi level, and a convenient notation of the scattering strength $c=\left(\pi N_{0} v_{0}\right)^{-1}$ is used.

\section{Conclusions}

In conclusion, we note a nonlocal suppression of the order parameter by the momentum-dependent term of the scattering potential and a strong on-site effect of the isotropic part of the impurity potential. Comparison of the isotropic and momentum-dependent impurity potentials shows that the anisotropy of the scattering potential leads to a weak suppression of the order parameter. Also the symmetry of the position-dependent order parameter is determined by the symmetry of the momentum-dependent impurity potential.

\section{References}

[1] P. Pisarski, G. Harań, Physica C 390, 270 (2003).

[2] A. Maciag, P. Pisarski, G. Harań, Acta Phys. Pol. B 34, 479 (2003).

[3] P. Pisarski, G. Harań, Phys. Status Solidi B 242, 426 (2005).

[4] P.W. Anderson, J. Phys. Chem. Solids 11, 26 (1959).

[5] L. Capriotti, D.J. Scalapino, R.D. Sedgewick, Phys. Rev. B 68, 014508 (2003).

[6] A.L. Fetter, Phys. Rev. 140, A1921 (1965).

[7] T. Xiang, J.M. Wheatley, Phys. Rev. B 51, 11721 (1995).

[8] G. Litak, Physica B 359-361, 566 (2005).

[9] A. Ghosal, M. Randeria, N. Trivedi, Phys. Rev. Lett. 81, 3940 (1998).

[10] G. Harań, A.D.S. Nagi, Phys. Rev. B 58, 12441 (1998). 